\title{
Chitin Based Fuel Cell and Its Proton Conductivity
}

\section{Takashi Kawabata, Yasumitsu Matsuo}

Department of Life Science, Faculty of Science \& Engineering, Setsunan University, Neyagawa, Osaka, Japan

Email: 17d901kt@edu.setsunan.ac.jp

How to cite this paper: Kawabata, T. and Matsuo, Y. (2018) Chitin Based Fuel Cell and Its Proton Conductivity. Materials Sciences and Applications, 9, 779-789. https://doi.org/10.4236/msa.2018.910056

Received: August 4, 2018

Accepted: September 3, 2018

Published: September 6, 2018

Copyright (c) 2018 by authors and Scientific Research Publishing Inc. This work is licensed under the Creative Commons Attribution International License (CC BY 4.0).

http://creativecommons.org/licenses/by/4.0/

\begin{abstract}
We have fabricated a fuel cell based on the tissue-derived biomaterial "chitin", and investigated its proton conductivity. It was found that chitin becomes the electrolyte of the fuel cell in the humidified condition, and power density of the fuel cell using chitin electrolyte becomes typically $1.35 \mathrm{~mW} / \mathrm{cm}^{2}$ at the $100 \%$ relative humidity. This result is the first result showing that the polysaccharide obtained from nature becomes the fuel cell electrolyte. Moreover, this result indicates that chitin is proton conductor in the humidified condition. In the chitin sheet plane, proton conductivity in chitin is observed approximately $0.1 \mathrm{~S} / \mathrm{m}$. Further, it was also found that chitin has the anisotropic proton conductivity. The proton conductivity along the chitin fiber direction is higher than that perpendicular to the chitin fiber direction. From these results, it is deduced that the formation of water bridges accompanied by hydration plays an important role in the appearance of proton conductivity in chitin.
\end{abstract}

\section{Keywords}

Fuel Cell, Proton Conductor, Electrolyte, Biomaterials

\section{Introduction}

As well-known, fuel cells attract attention as a clean next-generation energy. Among them, solid fuel cells are particularly attracting attention in terms of portability and stability. Therefore, these are required to find new electrolytes of the fuel cell, which is low cost and has no environmental load in the production process of the fuel cell. Recently, we focus on the tissue-derived biomaterials as the electrolyte of a fuel cell, because the tissue-derived biomaterials are low cost and abundant in nature. Chitin, which is one of tissue-derived bioma- 
terials, is obtained from the shells and tendon, such as crabs, shrimps, etc., and is biomass of huge amount in nature. Furthermore, the chitin and the deacetylate chitin (chitosan) have been recently used in the several fields such as the industrial and medical care field, because chitin and chitosan have superior strength, thermal stability and biocompatibility as the biomaterial. Malette et al. have investigated the curative effect of chitosan on the vulnerary [1]. Sandford et al. have suggested the useful substituent effect of chitosan to skin [2] [3]. Nair and Madhavan have investigated the method for the elimination of $\mathrm{Hg}$ in solution using chitosan [4]. Peniche-Covas et al. have investigated the efficiency of adsorption of $\mathrm{Hg}$ [5]. Jha et al. have also shown that chitosan powder has the effect on the adsorption of Cd ions [6]. Allan et al. reported that chitosan gives the wet strength of paper [7]. In the field of electrical device, Schulte et al. have shown that chitin can be used as an electrochemical sensor component [8]. In this way, a lot of investigations on chitin and chitosan have been carried out. This fact is realized by the characteristic features of chitin and chitosan which are easy to handle.

Recently, we have found that the fuel cell based on the biopolymer "collagen" electrolyte shows the maximum power density $0.86 \mathrm{~mW} / \mathrm{cm}^{2}$ with the humidified condition and that operates LED lighting as shown in Figure 1 [9]. This result indicates that tissue-derived biomaterials become the electrolyte of a fuel cell. Very recently, we have discovered the new bio-electrolyte of the fuel cell. This electrolyte consists of chitin, and the fuel cell based on chitin electrolyte exhibits higher power density compared with that based on other biomaterials, which are DNA and collagen and so on [9] [10]. Moreover, we have investigated the key factor in the appearance of proton conductivity in chitin. In the present paper, these results are shown. These results will be helpful for the development of further new fuel cells based on the biomaterials.

\section{Experimental}

\subsection{Sample Preparation}

Chitin films were prepared with the purified chitin obtained from crab shells (Sugino Machine Limited). This purified chitin slurry was well dispersed in distilled water and the chitin sheets were prepared by suction filtration. Figure 2 shows the photograph of the chitin film. The thickness of the film is approximately $0.07 \mathrm{~mm}$.

The chitin fiber specimens were prepared by purifying chitin obtained from the tendon of crab's legs. Based on the article of Prosky et al., the purification method was performed using a group of enzymes obtained from Streptomyces griseus (Wako Pure Chemical Industries, Ltd.) [11] [12] [13]. Figure 3 shows the purified the chitin fiber observed under the polarizing microscope. As shown in Figure 3, the extinction position and diagonal position are observed in the prepared chitin fiber. This result indicates that the purified chitin fiber has highly orientated structure. 


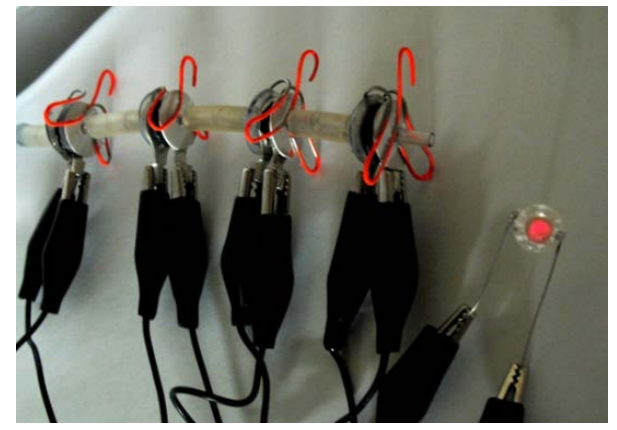

Figure 1. The fuel cell using collagen electrolyte is operating LED.

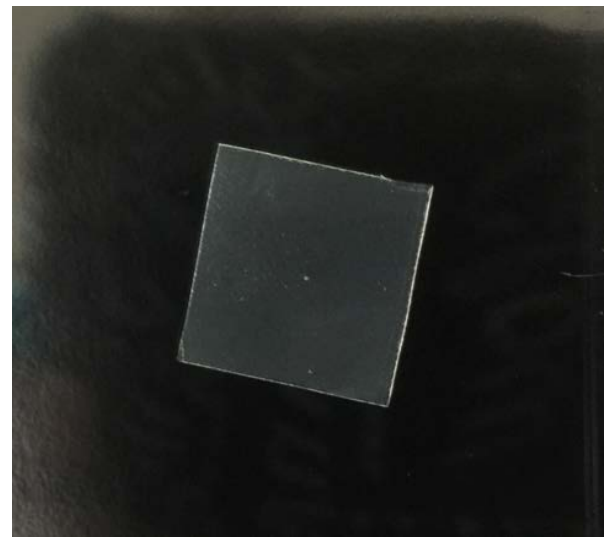

Figure 2. Photograph of chitin film.

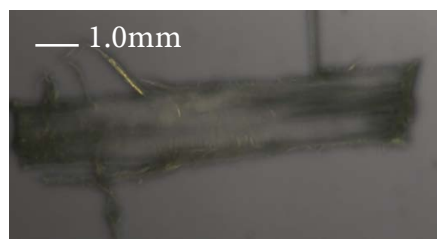

(a)

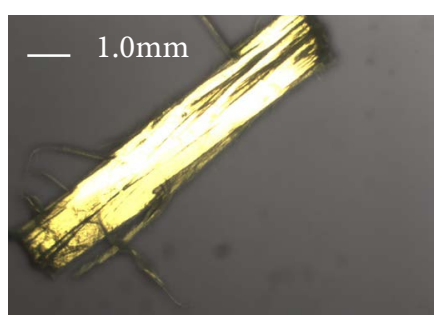

(b)

Figure 3. Chitin fiber: (a) extinction position and (b) diagonal position.

\subsection{Fabrication of the Fuel Cell Based on Chitin Electrolyte}

Figure 4 shows the shape of the fuel cell based on the chitin electrolyte. As show in Figure 4, the chitin electrolyte was inserted between Pt-C electrodes (anode and cathode). The current was collected from the current collector plates. The hydrogen and oxygen gases were introduced from the up and down sides of the fuel cell, respectively. In the fuel gas flow, the relative humidity, temperature and 
gas-flow ratio were controlled by the humidified gas-flow control system of Auto PEM (Toyo Corporation) at room temperature. The $\mathrm{H}_{2}$ gas flow rate and the air flow rate are $0.1 \mathrm{~L} / \mathrm{min}$ and $0.25 \mathrm{~L} / \mathrm{min}$, respectively.

\subsection{Impedance Measurement and Water Contents Measurement}

The water contents were measured from the relative humidity dependence of the weight of chitin using the electronic analytical balance (OHAUS Inc.) and the number of water molecules per a chitin molecule was calculated from the obtained water contents and molecular weights of water and mono-chitin.

The measurement of electrical conductivity was carried out using precision LCR meter (E4980A, Agilent Technologies Inc.). The relative humidity and temperature were controlled by the humidified gas-flow control system (Auto PEM). In the impedance measurement, the electrical conductivities perpendicular to the surface and parallel to the surface in chitin sheet were measured. In the case of chitin fiber specimens, impedance measurements were performed for specimens along the fiber direction and normal to the fiber direction, respectively.

\section{Results and Discussion}

Figure 5 shows the current density-cell voltage $\left(i-V_{\text {cell }}\right)$ curve of the fuel cell based on chitin electrolyte with the $100 \%$ relative humidity. As shown in Figure 5 , the cell voltage steeply decreases due to the anode over-potential at the very lower current region and then linearly decreases with increasing current density. In this way, the $i-V_{\text {cell }}$ curve shows the typical $i-V_{\text {cell }}$ characteristics of a fuel cell. This result indicates that the chitin becomes the electrolyte of the fuel cell and the chitin exhibits proton conductivity with the humidified condition. Further, it is also noted that the fuel cell using chitin electrolyte exhibits the maximum power density $1.35 \mathrm{~mW} / \mathrm{cm}^{2}$. This value is large compared with the fuel cell based on other biomaterials of collagen and DNA [10].

Figure 6 shows the relation between the relative humidity $R_{H}$ and $n$ in the humidified chitin. Here, $n$ is the number of water molecules per one molecule of the chitin at $300 \mathrm{~K}$. This result indicates that $n$ monotonically increases with the increase of humidity. This result indicates that there is no hydration-induced transition in the humidity region between $50 \%$ and $100 \%$ in chitin, and chitin has 2 water molecules per 1 molecule at the 100\% relative humidity. Therefore, for the analysis of proton conductivity, we can use not the relative humidity but the number " $n$ " as an index of the hydration.

Figure 7 shows the frequency dependence of AC conductivity. In Figure 7, AC conductivity is calculated with the equation $\sigma=\omega \varepsilon_{0} \varepsilon$ " obtained from the simple Capacitance-Resistance $(C-R)$ parallel equivalent circuit for specimen. Here, $\omega$ is angular frequency, $\varepsilon_{0}$ is the dielectric constant in vacuum, and $\varepsilon^{\prime \prime}$ is the imaginary part of dielectric constant. We can clearly see that all AC conductivity increases with the increase of the hydration number $n$. These results indicate that $\mathrm{AC}$ conductivity increases by the formation of the bonding between the 
chitin and water molecules. Moreover, it is noted that the conductivity calculated by $\sigma=\omega \varepsilon_{0} \varepsilon$ "strongly depends on the frequency. This result means that the chitin cannot be described by simple $C-R$ equivalent parallel circuit. In order to clear the origin of frequency dependence of AC electrical conductivity, we show the relation between the real and imaginary parts obtained from the measured impedance data in Figure 8. As shown in Figure 8, the observed impedance data $Z$ shows the semicircle part and linear parts in the impedance plane. This result indicates that two components are contained in the measured impedance. The almost linear behavior in impedance plane is well known as the contribution of Warburg impedance caused by the double layer capacitance and charge transfer resistance at the interface between the electrode and the specimen [14]. On the other hand, the part of semicircle behavior in impedance plane is also observed. When the specimen is considered by the simple $C-R$ parallel equivalent circuit, the impedance $Z$ can be described by the following equation,

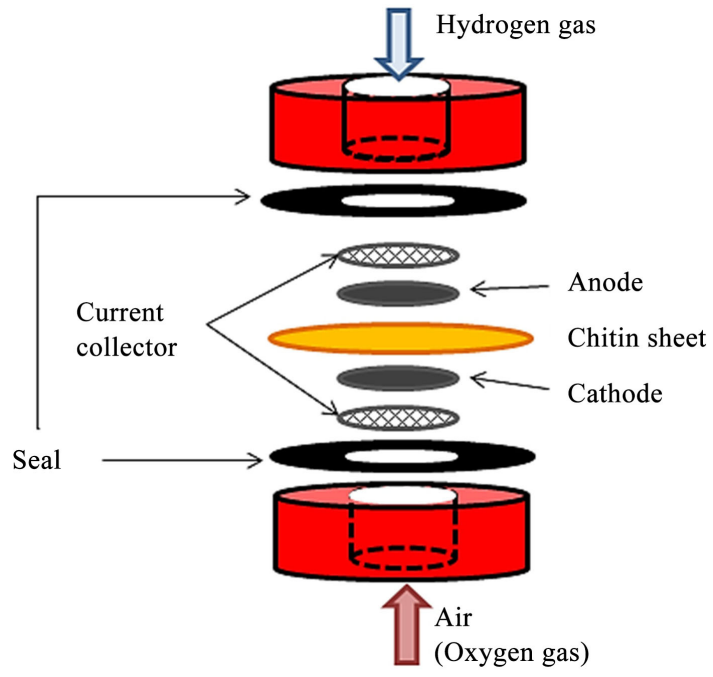

Figure 4. Fuel cell based on the chitin.

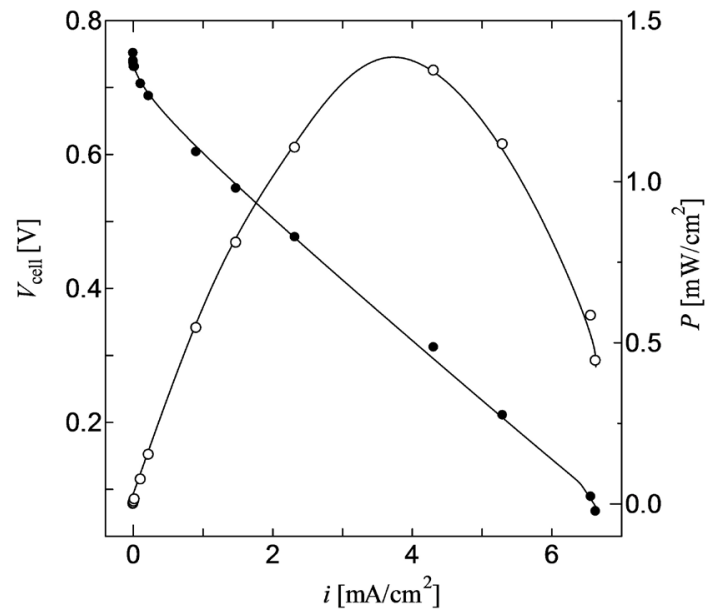

Figure 5. $i$ - $V$ curve of the fuel cell based on chitin electrolyte in the $100 \%$ relative humidity. 


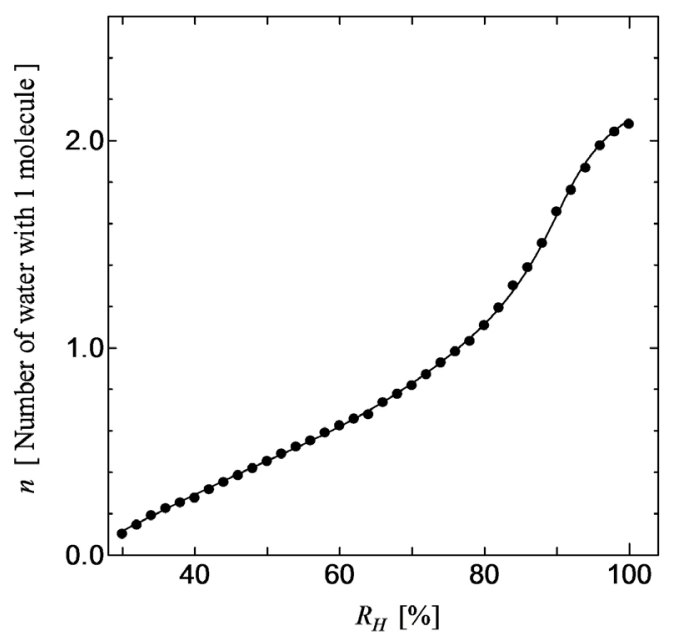

Figure 6. Water content of chitin with the humidification.

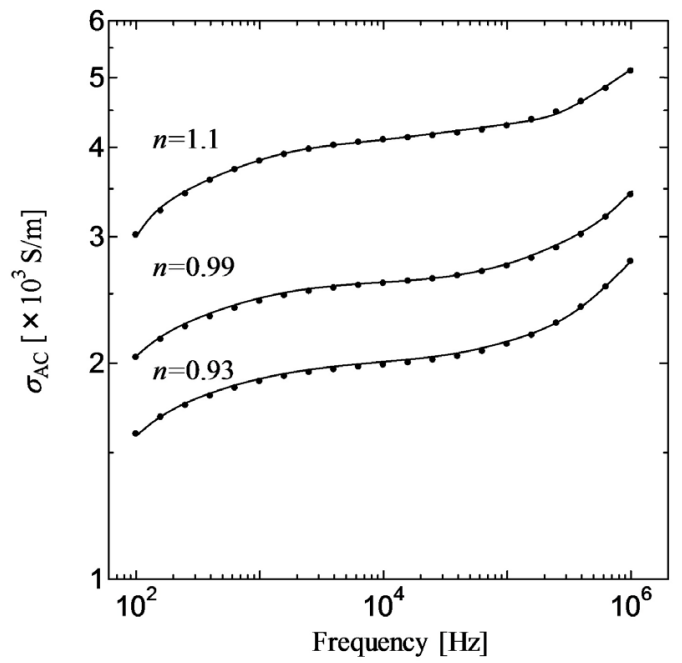

Figure 7. Frequency dependence of $\sigma_{\mathrm{AC}}$ at various $n$.

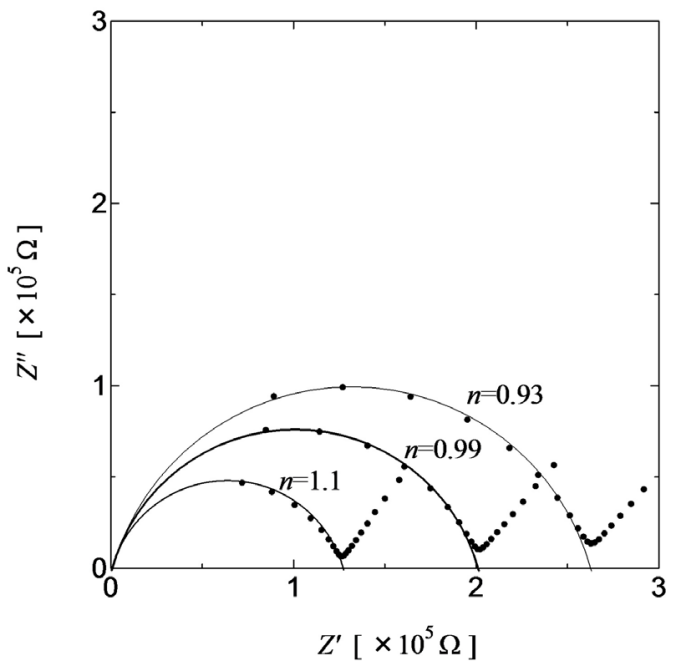

Figure 8. Relation between $Z$ 'and $Z$ ”in hydrated chitin. 


$$
Z=\frac{R}{1+(\omega C R)^{2}}-j \frac{\omega C R^{2}}{1+(\omega C R)^{2}}
$$

Here, $R$ and $C$ are resistance and capacitance in the simple $C$ - $R$ parallel equivalent circuit, respectively, and $j$ is imaginary unit.

For $Z^{\prime}=\frac{R}{1+(\omega C R)^{2}}$ and $Z^{\prime \prime}=j \frac{\omega C R^{2}}{1+(\omega C R)^{2}}$, the relation between $Z$ and $Z$ ' becomes the following equation,

$$
\left(Z^{\prime}-R / 2\right)^{2}+Z^{\prime 2}=(R / 2)^{2} .
$$

In this way, the semicircle part in the impedance plane is caused by the simple $C-R$ parallel equivalent circuit in the specimen. That is, considering these results, the observed semicircle part of $Z$ represents the conductivity and polarization property in bulk of chitin. In addition, we note that DC proton conductivity in the bulk of chitin can be derived by extrapolating the circular arc to the real axis in the impedance plane.

Figure 9 shows the relationship between DC proton conductivity $\sigma_{\mathrm{DC}}$ and $n$ in the bulk of chitin. The DC proton conductivity is obtained from the extrapolation of the circular arc using the method shown in Figure 8. As shown in Figure 9, DC proton conductivity increases with increasing $n$ and tends to saturate in the region of $n>1$. This result indicates that the formation of proton conduction path changes at around $n=1$. That is, the bonding of water molecules to chitin molecule at around $n=1$ changes from that below $n<1$. Considering that the bonding between chitin and water molecule is formed by hydrogen bond, the formation of hydrogen bond between chitin and water molecule plays an important role in proton conductivity. In addition, as shown in Figure 9, it is noted that there is the anisotropy for proton conductivity in chitin. It is evident that the water number $n$ dependences of $\sigma_{\mathrm{p}}$ and $\sigma_{\mathrm{N}}$ show the similar behavior. Moreover, it is also noted that $\sigma_{\mathrm{p}}$ becomes approximately $1 \times 10^{-1} \mathrm{~S} / \mathrm{m}$ at $n=2$. On the other hand, $\sigma_{\mathrm{N}}$ becomes about $2 \times 10^{-2} \mathrm{~S} / \mathrm{m}$. The conductivity normal to plane is 5 times smaller than that in plane. These results indicate that the direction of bonding between the chitin and water molecules is important for the determination of the value of proton conductivity. It is well known that the chitin has fiber structure. Therefore, in order to investigate the orientational dependence of proton conductivity in detail, we have measured the difference between proton conductivities along the fiber $\sigma_{\mathrm{PF}}$ and perpendicular to the fiber $\sigma_{\mathrm{NF}}$.

Figure 10 shows the humidity dependences of $\sigma_{\mathrm{PF}}$ and $\sigma_{\mathrm{NF}}$. Here, $\sigma_{\mathrm{PF}}$ and $\sigma_{\mathrm{NF}}$ are proton conductivity parallel and perpendicular to chitin fiber, respectively. It is evident that $\sigma_{\mathrm{PF}}$ has approximately 6 times higher than $\sigma_{\mathrm{NF}}$. In the sheet sample in Figure 9, it is deduced that chitin fiber is lied in plane of chitin sheet and that therefore the conductivity in plane is mainly related to proton conductivity along the fiber. On the other hand, proton conductivity normal to the chitin sheet is mainly contributed by that perpendicular fiber direction. Therefore, the anisotropy for proton conductivity of chitin fiber in Figure 10 is consistent with 
that in sheet sample (Figure 9). Considering the results for anisotropic proton conductivity, it appears that the relation between chitin hydrated structure and proton conduction path formed by the hydrogen bond with water molecule is significantly important.

Figure 11 shows the crystal structures in the $a-c$ and $b-c$ planes in the hydrated chitin reported by many researchers [15]-[20]. As shown in Figure 11(a), it is evident that the hydrated chitin has water network which is formed along the chitin fiber direction. On the contrary, as shown in Figure 11(b), the formation of water network perpendicular to the chitin fiber direction is difficult compared with that along the fiber direction, because water molecules are bonded between chitin fibers. Therefore, proton transport in perpendicular to the fiber direction is prevented by difficulty of formation of the water network. These results indicate that the direction of water network is closely related to the value of proton conductivity. It is also noted that these water molecules form hydrogen bonds between hydroxyl group and amino-acetyl group in chitin, or between water molecule and next water molecule. Considering that proton conductivity increases with the increase in the number of water molecule and that proton conductivity along the direction of water network becomes high, these results indicate that the realization of proton conductivity is achieved by proton transfer via the water network formed between chitin and water molecules with the breaking and rearrangement of hydrogen bond. In addition, it seems that the hydroxyl group and amino-acetyl group in the side chain of chitin, which form the hydrogen bonds with water molecules, facilitate the generation of hydronium ions. It is deduced that the generated hydronium ions in the water network move with the breaking and rearrangement of hydrogen bond, and consequently proton conductivity is realized in the water network. It is necessary to investigate the quantitative analyses between the water network and proton conductivity, in

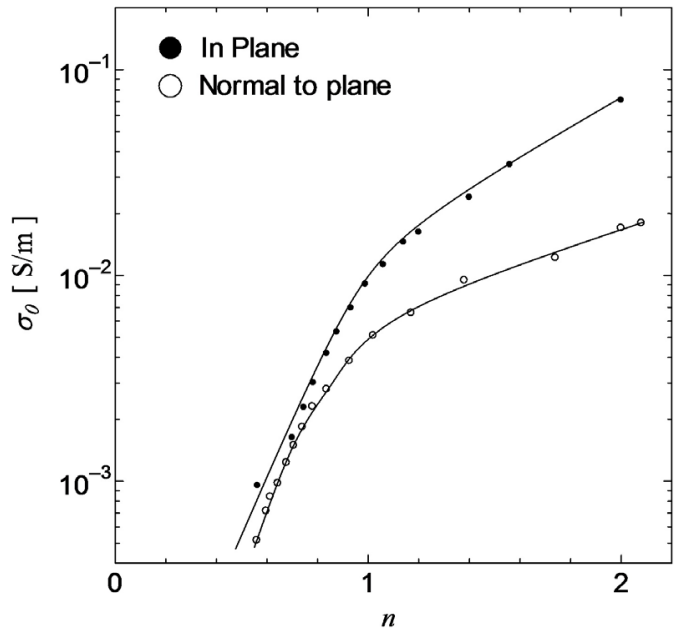

Figure 9. Relation between hydration number and proton conductivity in chitin sheet. Solid circle and open circle denote proton conductivity in plane of chitin sheet and normal to chitin sheet, respectively. 


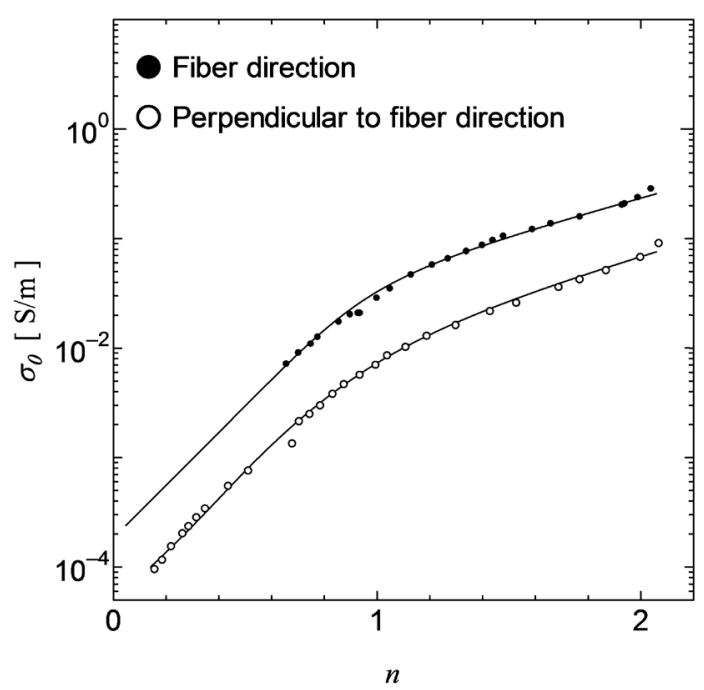

Figure10. Orientation-dependent proton conductivity in orientation chitin.

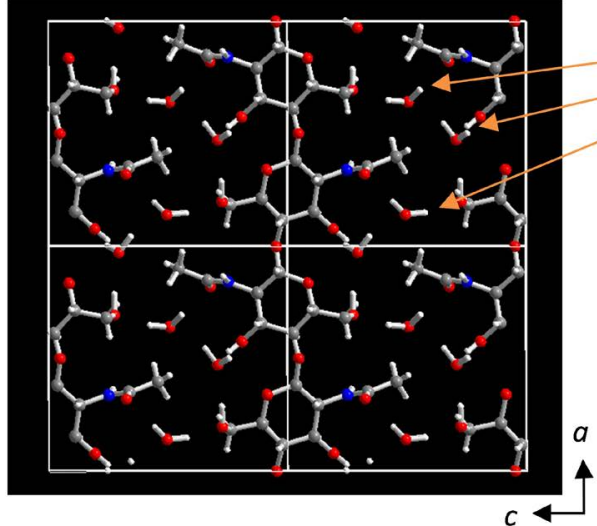

(a)

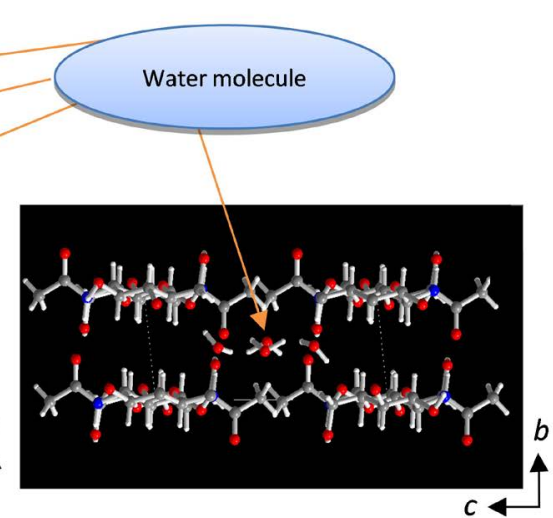

(b)

Figure 11. Structure of hydrated chitin [18] [19] [20]. (a) in the $a-c$ plain and (b) in the $b-c$ plane. These pictures show ball and stick model of hydrated chitin. Gray, red, blue and white ball show carbon, oxygen, nitrogen and hydrogen atoms.

order to understand the anisotropic conductivity in chitin in detail. These results will appear future issues.

\section{Conclusion}

We have fabricated the chitin fuel cell and investigated whether the chitin becomes the electrolyte or not. As a result, chitin becomes proton conductor and operates as the electrolyte of the fuel cell. The proton conductivity increases with the increase in the number of water molecule. Moreover, chitin has the anisotropic conductivity from the measurement of proton conductivity in the sheet and highly-orientated specimens. It is found that proton conductivity along the chitin fiber direction becomes higher than that perpendicular to the chitin fiber direction. Considering that water molecules is bonded with the side chain of chitin along the chitin fiber direction, the appearance of proton conductivity in chi- 
tin is caused by the proton transfer via the water network formed between the chitin and water molecules.

\section{Conflicts of Interest}

The authors declare no conflicts of interest regarding the publication of this paper.

\section{References}

[1] Malettas, W.G., Quingley, H.J. and Adickes, E.D. (1986) Chitin in Nature and Technology. Plenum Press, New York, 435.

[2] Olsen, R., Schwartzmiller, D., Weppner, W. and Winandy, R. (1989) Biomedical Application of Chitin and Its Derivates, in Chitin and Chitosan. Sources, Chemistry, Biochemistry, Physical Properties and Applications. Elsevier Applied Science, New York.

[3] Sandford, P.A. and Stinnes, A. (1991) Biomedical Applications of High Purity Chitosan; Physical, Chemical and Bioactive Properties. ACS Symposium Series, 467, 430 .

[4] Nair, K.G.R. and Madhavan, P. (1984) Chitosan for Removal of Mercury from Water. Fishery Technology, 21, 109-112.

[5] Peniche-Covas, C., Alwarez, L.W. and Arguelles-Monal, W. (1992) The Adsorption of Mercuric Ions by Chitosan. Journal of Applied Polymer Science, 46, 1147-1150. https://doi.org/10.1002/app.1992.070460703

[6] Jha, I.N., Leela, I. and Prabhakar Rao, A.V.S. (1988) Removal of Cadmium Using Chitosan. Journal of Environmental Engineering, 114, 962. https://doi.org/10.1061/(ASCE)0733-9372(1988)114:4(962)

[7] Allan, G., Crospy, G.D., Lee, J.H., Miller, M.L. and Reif, W.M. (1972) New Bonding Systems for Paper. Proceedings of a Symposium on Man-Made Polymers in Paper Making, Helsinki, 5-8 June 1972, 85-95.

[8] Suginta, W., Khunkaewla, P. and Schulte, A. (2013) Electrochemical Biosensor Applications of Polysaccharides Chitin and Chitosan. Chemical Reviews, 113, 5458-5479. https://doi.org/10.1021/cr300325r

[9] Matsuo, Y. (2014) Bio-Fuel Cell Based on Biopolymer Electrolyte. The Journal of Fuel Cell Technology, 13, 60.

[10] Matsuo, Y., Kumasaka, G., Saito, K. and Ikehata, S. (2005) Fabrication of Solid-State Fuel Cell Based on DNA Film. Solid State Communications, 133, 61-64. https://doi.org/10.1016/j.ssc.2004.09.055

[11] Prosky, L., Asp, N.G., Furda, I., Devries, J.W., Schweizer, T.F. and Harland, B.F. (1984) Determination of Total Dietary Fiber in Foods, Food Products and Total Diets. Journal-Association of Official Analytical Chemists, 67, 1044-1052.

[12] Prosky, L., Asp, N.G., Furda, I., Devries, J.W., Schweizer, T.F. and Harland, B.F. (1985) Determination of Total Dietary Fiber in Foods and Food Products: Collaborative Study. Journal-Association of Official Analytical Chemists, 68, 677-679.

[13] Maezaki, Y., Yamazaki, A., Mizuochi, K. and Tsuji, K. (1993) Measurement of Dietary Fiber in Chitin and Chitosan by the Enzymatic-Gravimetric Metho. Journal of the Agricultural Chemical Society of Japan, 67, 677-684. https://doi.org/10.1271/nogeikagaku1924.67.677

[14] Katayama, H. (2014) Surface and Interfacial Analysis Using Electrochemical Im- 
pedance Measurement. Journal of the Japan Institute of Metals and Materials, 78, 419-425. https://doi.org/10.2320/jinstmet.JB201402

[15] Okuyama, K., Noguchi, K. and Miyazawa, T. (1997) Molecular and Crystal Structure of Hydrated Chito. Macromolecules, 30, 5849-5855.

https://doi.org/10.1021/ma970509n

[16] Sikorski, P., Hori, R. and Wada, M. (2009) Revisit of $\alpha$-Chitin Crystal Structure Using High Resolution X-Ray Diffraction Data. Biomacromolecules, 10, 1100-1105. https://doi.org/10.1021/bm801251e

[17] Kobayashi, K., Kimura, S., Togawa, E., Wada, M. and Kuga, S. (2010) Crystal Transition of Paramylon with Dehydration and Hydration. Carbohydrate Polymers, 80, 491-497. https://doi.org/10.1016/j.carbpol.2009.12.009

[18] Nishiyama, Y., Noishiki, Y. and Wada, M. (2011) X-Ray Structure of Anhydrous $\beta$-Chitin at 1 Å Resolution. Macromolecules, 44, 950-957. https://doi.org/10.1021/ma102240r

[19] Sawada, D., Nishiyama, Y., Langan, P., Forsyth, V.T., Kimura, S. and Wada, M. (2012) Water in Crystalline Fibers of Dihydrate $\beta$-Chitin Results in Unexpected Absence of Intramolecular Hydrogen Bonding. PLoS ONE, 7, e39376. https://doi.org/10.1371/journal.pone.0039376

[20] Sawada, D., Nishiyama, Y., Langan, P., Forsyth, V.T., Kimura, S. and Wada, M. (2012) Direct Determination of the Hydrogen Bonding Arrangement in Anhydrous $\beta$-Chitin by Neutron Fiber Diffraction. Biomacromolecules, 13, 288-291. https://doi.org/10.1021/bm201512t 